\title{
Extended Retrosigmoid Approach for the Resection of a Pontomedullary Junction Cavernous Malformation
}

\author{
Jaafar Basma ${ }^{1} \quad$ Vincent Nguyen $^{1} \quad$ Jeffrey Sorenson ${ }^{1} \quad$ L. Madison Michael II \\ ${ }^{1}$ Department of Neurosurgery, University of Tennessee, Memphis, \\ Tennessee, United States \\ J Neurol Surg B 2018;79(suppl S5):S418-S419.

\begin{abstract}
Address for correspondence L. Madison Michael II, MD, Department of Neurosurgery, University of Tennessee, 847 Monroe Avenue, Suite 427, Memphis, TN 38163, United States
\end{abstract} \\ (e-mail: mmichael@semmes-murphey.com).
}

\begin{abstract}
Objectives To describe an extended retrosigmoid approach for the resection of a cavernoma involving the ponto-medullary junction, with emphasis on the microsurgical anatomy and technique.

Design A retrosigmoid craniotomy is performed in the lateral decubitus position and the sigmoid sinus exposed. After opening the dura, sutures are placed medial to the sinus to allow its gentle mobilization. Cerebrospinal fluid (CSF) is drained from the cisterna magna, and cerebellopontine cistern, and dynamic retraction is used over the cerebellum. Subarachnoid dissection of the cerebellopontine angle gives access to cranial nerves IX/X, VII/VIII, and VI. Inspection of the pontomedullary junction medial to the facial nerve reveals hemosiderin staining in that region. A small pial opening is made, exposing the hemorrhagic cavity. The cavernous malformation is then identified, dissected circumferentially, and resected. Photographs of the region are borrowed from Dr Rhoton's laboratory to illustrate the microsurgical anatomy.

\section{Keywords}

- cerebellopontine angle

- cavernous malformation

- brainstem

- pontomedullary junction

- microsurgery

- retrosigmoid approach

Participants The senior author performed the surgery. The video was edited by Drs. J.B. and V.N.

Outcome Measures Outcome was assessed with extent of resection and postoperative neurological function.

Results A gross total resection of the lesion was achieved. The patient did not develop any postoperative deficits.

Conclusion Understanding the microsurgical anatomy of the cerebellopontine angle and meticulous microneurosurgical technique are necessary to achieve a complete resection of a brainstem cavernoma. The extended retrosigmoid approach provides an adequate corridor to the pontomedullary junction.

The link to the video can be found at: https://youtu.be/FIKixWJT75w.
\end{abstract}

Conflict of Interest

None.

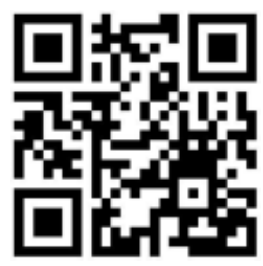

received

May 31, 2018

accepted

August 11, 2018

published online

September 25, 2018 www.thieme.com/skullbasevideos

www.thieme.com/jnlsbvideos
License terms

10.1055/s-0038-1669979.

ISSN 2193-6331. (c) 2018 Georg Thieme Verlag KG

Stuttgart · New York (c) $\odot \ominus$ 

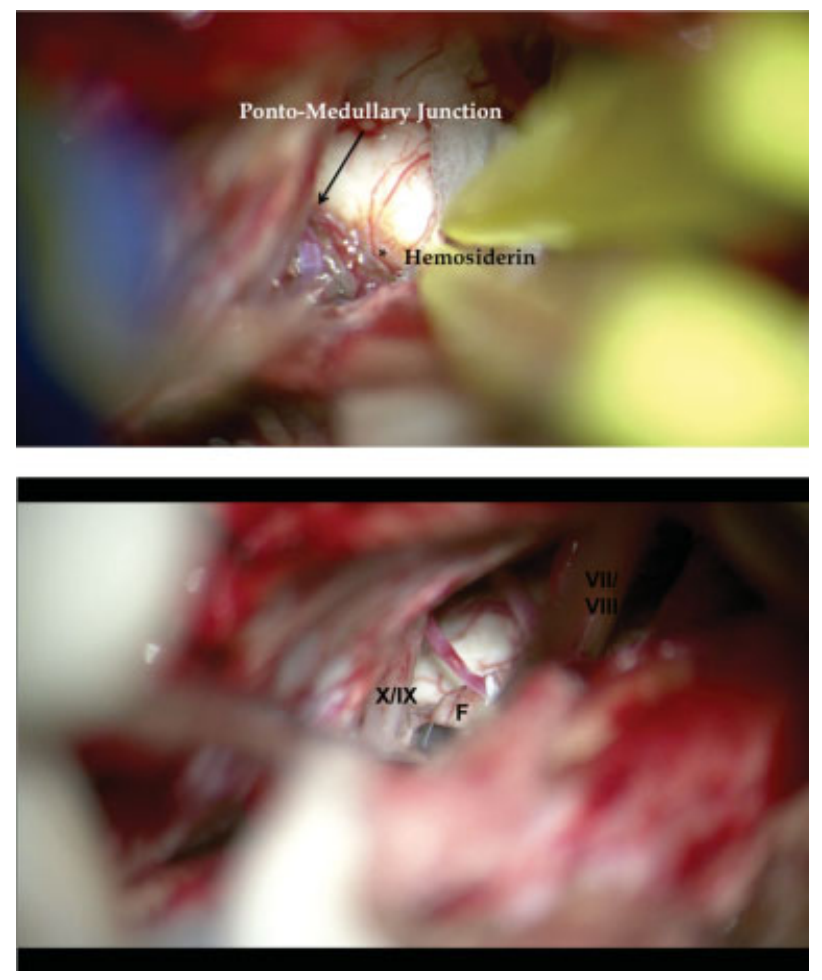

Fig. 1 The cavernous malformation is visualized at the surface of the pontomedullary junction with hemosiderin staining. Access to the lesion requires delicate exposure of the lower cranial nerves as detailed in this figure.
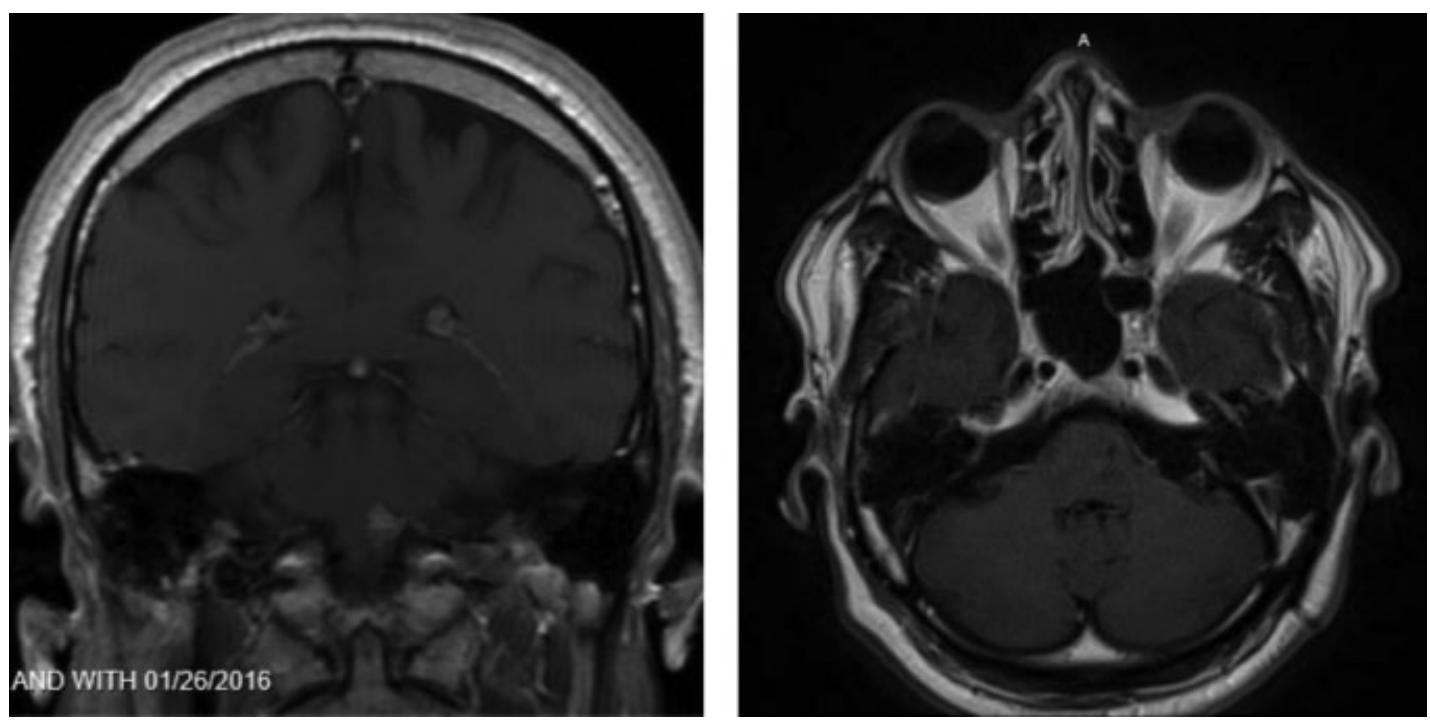

Fig. 2 The cavernous malformation is seen clearly at the pontomedullary junction on this coronal magnetic resonance imaging (MRI). Postoperative contrasted imaging shows gross total resection of the lesion. 